\title{
EDITORIAL
}

\section{Special issue on Cyberworlds'2007}

\author{
Nadia Magnenat-Thalmann • Franz-Erich Wolter
}

Published online: 13 August 2008

(C) Springer-Verlag 2008

The sixth International Conference on Cyberworlds (Cyberworlds 2007) took place in the beautiful town of Hannover, Germany, in October 2007. The HAPTEX Workshop 2007 was co-hosted with the conference.

The first workshop on Cyberworlds was held as the International Workshop on Synthetic Worlds in the University of Aizu, Japan, in 1993, to explore the meaning, philosophy, and the potential of the worlds synthesized on the web as well as in computational spaces, in general. Since then, the conferences are organized yearly. The HAPTEX workshop is the second workshop held under the project "HAPtic sensing of virtual TEXtiles" (HAPTEX). The project is funded under the Sixth Framework Programme (FP6) of the European Union and is coordinated by Prof. Nadia Magnenat-Thalmann who heads MIRALab at the University of Geneva, Switzerland.

Five best papers from the Cyberworlds conference and two best papers from the HAPTEX workshop have been selected for this special issue of The Visual Computer. The papers have been extended and carefully revised for the journal.

The papers reflect some of the diverse subject areas included under the banner of cyberworlds and haptic interaction. We start with "Real-Time Crowd Motion Planning: Scalable Avoidance and Group Behavior" which deals with the difficult task of real-time motion planning for large crowds while ensuring short-term collision avoidance and efficient group behavior. We then present "Function-based visualization and haptic rendering in shared virtual spaces" which shows how to define tangible physical properties of the objects, together with their geometry and appearance, by using mathematical functions. We then move on to a scalable platform for designing distributive, multi-player 3D games in "A model for adapting 3D graphics based on scalable coding, real-time simplification, and remote rendering." "Automatic Spatial Analysis and Pedestrian Flow Control for Real-time Crowd Simulation in an Urban Environment" presents a technique to distribute pedestrian populations in an urban environment. "Multilayered Visuo-Haptic Hair Simulation," uses a two-layered model to enable real-time visual and haptic interaction with hair. We continue with "Haptic Two-Finger Contact with Textiles" which presents a haptic simulation system that allows the user to interact with a virtual fabric using two fingers while maintaining the accuracy required for haptic and physically-based simulation. The problem of perception in virtual environments under crossmodal interactions is tackled in "Visual-Haptic Perception of Compliant Objects in Artificially Generated Environments."

Program Co-chairs Cyberworlds 2007 and HAPTEX Workshop 2007

N. Magnenat-Thalmann $(\bowtie)$

MIRALab, Geneva, Switzerland

e-mail: thalmann@miralab.unige.ch

F.-E. Wolter

Leibniz Universität Hannover, Hanover, Germany 